\title{
Mineralocorticoid receptor blockade improves glucocorticoid-induced skin atrophy but partially ameliorates anti-inflammatory actions in an irritative model in human skin explants
}

Julia Boix ${ }^{1}$, Van Tuan Nguyen ${ }^{2,3}$, Nicolette Farman ${ }^{2}$, Sélim Aractingi ${ }^{4}$, Paloma Pérez ${ }^{1 *}$

${ }^{1}$ Instituto de Biomedicina de Valencia, Consejo Superior de Investigaciones Científicas

(IBV-CSIC), Valencia, Spain

${ }^{2}$ UMR 1138, INSERM, Centre de Recherche des Cordeliers, Paris, France

${ }^{3}$ Basic Science Department, Thai Nguyen University of Agriculture and Forestry, Thai Nguyen, Vietnam

${ }^{4}$ Hôpital Cochin Tarnier, Département de Dermatologie and Université Paris Descartes, Paris, France

* Corresponding author: Paloma Pérez, IBV-CSIC, Jaime Roig 11, E-46010-Valencia, Spain. Tel +34963391766 Fax +34963690800

pperez@ibv.csic.es

Short title: MR blockade improves GC-induced skin atrophy in human damaged skin

Keywords: Mineralocorticoid receptor, skin, atrophy, glucocorticoids, damage

Word count: 1044

\section{Funding}

This work was supported by the Spanish Ministerio de Ciencia e Innovación (grant SAF201459474-R) and by the ADMIRE COST Action BM-1301. 


\begin{abstract}
We recently demonstrated that blockade of the mineralocorticoid receptor (MR) effectively ameliorated GC-induced skin atrophy in healthy human skin explants and epidermal MR knockout mice. However, whether MR blockade improves the therapeutic index of GCs in skin pathology was not investigated.

We assessed the effects of GCs, MR antagonists (MRA), or both, in SDS-treated human skin explants. All treatments restored SDS-augmented epidermal thickness but only GC plus MRA restored the expression of COL1A1. However, MRA alone or in combination with GCs may exert a dual role in regulating inflammatory cytokines. Thus, although combined treatment may be beneficial to improve irritative skin, extensive in vivo testing is required to establish whether the anti-inflammatory effects of GCs are maintained in the presence of MRA.
\end{abstract}




\section{Background}

Skin inflammatory diseases are commonly treated with topical glucocorticoids (GCs) because their anti-proliferative and anti-inflammatory effects (1). However, their use is limited due to adverse side effects such as skin atrophy characterized by tissue thinning caused by inhibition of keratinocyte and fibroblast proliferation and a reduction in the collagen synthesis $(1,2)$. Also, local steroidogenic activity is key to homeostatic prevention or attenuation of skin pathology $(3,4)$.

GCs bind and activate two closely related receptors, the GC receptor (GR) and the mineralocorticoid receptor (MR). Our recent work using genetic and pharmacological approaches in mouse skin and healthy skin of volunteers and human skin explants has shown that MR inactivation ameliorates GC-induced skin atrophy $(5,6)$. In both settings, MR blockade improved the epidermal thinning partially reversing the keratinocyte proliferation inhibition induced by topical GC treatments $(5,6)$. However, GCs similarly reduced Collal mRNA levels in MR epidermal knock-out and control mice indicating that inactivation of dermal MR is also necessary for the effective reversion of skin atrophy (5).

\section{Questions addressed}

We have analyzed whether GC treatment combined with pharmacological MR inhibition achieves a better therapeutic index in an irritative model in human skin explants.

\section{Experimental design}

Human skin explants culture and treatments

Human skin biopsies were obtained from three different subjects. After adipose tissue excision, $6 \mathrm{~mm}$ full-thickness punches were obtained of each subject, placed in organotypic culture floating with the dermis embedded into the medium, and used for one independent experiment. Human skin explants were cultured in medium DMEM 1x with GlutaMAX, 10\% charcoalstripped fetal bovine serum and $1 \%$ Penicilin/Streptomicin, and incubated at $37^{\circ} \mathrm{C}, 5 \% \mathrm{CO}_{2}(6)$. SDS (15 $\mu \mathrm{g} / \mathrm{ml}$; Merck, Darmstadt, Germany) was added to the medium during 3 days; then, washed, and explants were treated with $100 \mathrm{nM}$ clobetasol propionate, $10 \mu \mathrm{M}$ canrenoate, 100 $\mathrm{nM}$ clobetasol plus $10 \mu \mathrm{M}$ canrenoate (Sigma, St Louis, MO), or vehicle, during 4 days.

\section{Results}

Using human skin explants, we have mimicked an irritative pathology by adding SDS to the medium (7). After 3 days, SDS-induced tissue damage was revealed in hematoxylin-eosin stained sections by cell ballooning (Fig.1a, arrows), increased dermal cellularity (Fig.1a) and augmented epidermal thickness (Fig.1a,b). These features were similar to those observed in human skin topically treated with SDS $(8,10)$. However, SDS did not affect collagen deposition or mRNA expression levels relative to vehicle-treated samples (Fig.s1a,b).

We assessed the therapeutic effectiveness of the GC clobetasol, the MRA canrenoate, or both, in skin explants incubated with SDS. Clobetasol improved the SDS-induced effects by reducing 
cell ballooning, dermal cellularity, and epidermal thickness (Fig.1a,b). As expected, clobetasol also reduced collagen deposition as shown by the Masson trichrome staining and the $70 \%$ reduction in COLIA1 mRNA levels (Fig.s1a,b). Treatment with canrenoate elicited similar effects than GCs in terms of epidermal thickness and COL1Al expression, with a reduction of approximately $60 \%$ in both parameters (Fig.1a,b; s1a,b). This is consistent with previous data reporting that the induced expression of COL1Al in human dermal fibroblast was effectively blocked by treatment with the MRA spironolactone (9). Importantly, the combined treatment using clobetasol and canrenoate was also effective in decreasing the SDS-induced epidermal thickening (Fig.1a,b). In addition, this treatment was the only that improved the collagen deposition and the COL1A1 mRNA levels as compared to each compound used separately (Fig. s1a,b).

We also evaluated the expression of keratin $(\mathrm{K}) 6$, a marker of keratinocyte hyperproliferation that is found in healthy palmar and plantar skin as well as diseased skin, in explants treated with SDS alone or in combination with the indicated compounds. After SDS treatment, all epidermal layers stained positive for K6; this expression was significantly reduced by clobetasol, which was confined to the upper epidermal layers, but unaffected by canrenoate (Fig.2a). The combined treatment reduced the effectiveness of clobetasol alone as it produced an intermediate effect with K6 being expressed in several suprabasal layers (Fig.2a). These results suggest that complete K6 inhibition by GCs requires functional MR.

Next, we assessed whether the anti-inflammatory effects of GCs were preserved after blocking MR. We analyzed the expression of the pro-inflammatory cytokines $I L-6$ and $I L I A$ and a key enzyme in prostaglandin biosynthesis, $C O X-2$, which are up-regulated after SDS treatment in human keratinocytes $(11)$ or skin $(8,12)$. Clobetasol significantly reduced the expression of SDS-induced $I L-6, I L 1 A$, and $C O X-2$ mRNA levels, as expected (Fig.2b). However, canrenoate triggered both anti-inflammatory effects, with decreased expression of $I L-6$ and $C O X-2$, and also pro-inflammatory, with up-regulation of ILIA mRNA levels (Fig.2b). The use of clobetasol plus canrenoate resulted in attenuated or lack of repression of $I L-6$ and $I L I A$, respectively, while it had no effect in the inhibition of $C O X-2$ relative to clobetasol alone (Fig.2b). Also, consistent with its reported induction by GCs (13), Clobetasol up-regulated the mRNA levels of the antiinflammatory cytokine $I L-10$ in SDS-treated skin explants (Fig. 2b). While canrenoate did not affect $I L-10$ mRNA levels, clobetasol plus canrenoate resulted in a trend towards increased transcript levels without reaching statistical significance, suggesting that MR blockade affects GC-dependent induction of $I L-10$. These results show that MR may exert a dual role in skin inflammation.

\section{Conclusions}

SDS-treatment in human skin explants induced tissue damage as demonstrated by cell ballooning, increased epidermal thickening and dermal cellularity. In this clinically relevant setting, MRA alone was as effective as GCs in normalizing epidermal thickness although it was unable to control up-regulation of $\mathrm{K} 6$ expression and exerted dual pro- and anti-inflammatory actions. The combined use of GCs plus MRA had several advantages relative to individual treatments since it restored epidermal thickness, partially reversed K6 up-regulation, and normalized collagen deposition. However, in this model of human diseased skin, the antiinflammatory effects of GCs, in the case of IL6 and ILIA, were not fully preserved in the 
presence of MRAs. Overall, our data show that addition of MRA to GCs may be beneficial to improve irritative skin, but extensive in vivo testing is required to establish whether the antiinflammatory effects of GCs are maintained in the presence of MRA.

\section{Acknowledgements}

We thank Jose Nieto for expert technical help. JB is recipient of FPI fellowships from MINECO (SAF2014-59474-R). We thank the COST ADMIRE BM-1301 for financial support and dissemination of the results. JB and VTN performed the research; NF, SA, and PP designed the experiments; JB and PP drafted the paper; all authors analyzed the data and critically revised the manuscript.

\section{Conflict of interest}

The authors have declared no conflict of interests.

\section{References}

1 Schoepe S, Schäcke H, May E \& Asadullah K 2006 Glucocorticoid therapy-induced skin atrophy. Exp Dermatol 15(6):406-420.

2 Schäcke H, Berger M, Rehwinkel H \& Asadullah K 2007 Selective glucocorticoid receptor agonists (SEGRAs): novel ligands with an improved therapeutic index. Mol Cell Endocrinol 275(1-2):109-117.

3 Slominski AT, Manna PR \& Tuckey RC 2014 Cutaneous glucocorticosteroidogenesis: securing local homeostasis and the skin integrity. Exp Dermatol 23(6):369-374.

4 Slominski AT, Manna PR \& Tuckey RC 2015 On the role of skin in the regulation of local and systemic steroidogenic activities. Steroids 103:72-88.

5 Boix J, Sevilla LM, Sáez Z, Carceller E \& Pérez P 2016 Epidermal Mineralocorticoid Receptor Plays Beneficial and Adverse Effects in Skin and Mediates Glucocorticoid Responses. J Invest Dermatol 136(12):2417-2426.

6 Maubec E, Laouénan C, Deschamps L, Nguyen VT, Scheer-Senyarich I, WackenheimJacobs AC, Steff M, Duhamel S, Tubiana S, Brahimi N, Leclerc-Mercier S, Crickx B, Perret C, Aractingi S, Escoubet B, Duval X, Arnaud P, Jaisser F, Mentré F \& Farman N 2015 Topical Mineralocorticoid Receptor Blockade Limits Glucocorticoid-Induced Epidermal Atrophy in Human Skin. J Invest Dermatol 135(7):1781-1789.

7 Le TK, De Mon P, Schalkwjik J \& van der Valk PG 1997 Effect of a topical corticosteroid, a retinoid and a vitamin D3 derivative on sodium dodecyl sulphate induced skin irritation. Contact Dermatitis 37(1):19-26.

8 Clemmensen A, Andersen KE, Clemmensen O, Tan Q, Petersen TK, Kruse TA \& Thomassen M 2010 Genome-wide expression analysis of human in vivo irritated epidermis: differential profiles induced by sodium lauryl sulfate and nonanoic acid. J Invest Dermatol 130(9):2201-2210.

9 Mitts TF, Bunda S, Wang Y \& Hinek A 2010 Aldosterone and mineralocorticoid receptor antagonists modulate elastin and collagen depositionin human skin. J Invest Dermatol 130(10):2396-2406. 
10 Varani J, Perone P, Spahlinger DM, Singer LM, Diegel KL, Bobrowski WF \& Dunstan R 2007 Human skin in organ culture and human skin cells (keratinocytes and fibroblasts) in monolayer culture for assessment of chemically induced skin damage. Toxicol Pathol 35(5):693-701.

11 Oh J, Yu T, Choi SJ, Yang Y, Baek HS, An SA, Kwon LK, Kim J, Rho HS, Shin SS, Choi WS, Hong S \& Cho JY 2012 Syk/Src pathway-targeted inhibition of skin inflammatory responses by carnosic acid. Mediators Inflamm 2012:781375-781388.

12 Coquette A, Berna N, Vandenbosch A, Rosdy M, De Wever B \& Poumay Y 2003 Analysis of interleukin-1alpha (IL-1alpha) and interleukin-8 (IL-8) expression and release in in vitro reconstructed human epidermis for the prediction of in vivo skin irritation and/or sensitization. Toxicol In Vitro 17(3):311-21.

13 Coutinho AE, Chapman KE 2011 The anti-inflammatory and immunosuppressive effects of glucocorticoids, recent developments and mechanistic insights. Mol Cell Endocrinol. $335(1): 2-13$. 


\section{Figure legends}

Figure 1. Combined treatment using GCs (Clobetasol) and MR antagonists (Canrenoate) in human skin explants confers an overall protection against SDS-induced skin damage.

a) H\&E staining of human skin explants treated with vehicle only or SDS plus vehicle (V), clobetasol (Clobe), canrenoate (Canre), or Clobe+Canre. Broken lines separate epidermis from dermis. Arrows indicate cell ballooning of suprabasal keratinocytes induced by SDS. Bar: $50 \mu \mathrm{m}$.

b) Epidermal thickness quantitation was assessed in the same groups as in a). ANOVA, post hoc Tukey test: $\mathrm{n}=3$ of each treatment, $* * * \mathrm{p}<0.001$, differences versus vehicle only; \#\#\# $\mathrm{p}<0.001$, differences versus SDS+V; $+\mathrm{p}<0.05$, differences between other treatments.

Figure 2. Epidermal damage induced by SDS in human skin explants is not completely restored by Clobetasol upon MR pharmacological blockade.

a) Immunohistochemistry for K6 of human skin explants treated with SDS plus vehicle (V), clobetasol (Clobe), canrenoate (Canre), or Clobe+Canre. Broken lines separate epidermis from dermis. Bar: $50 \mu \mathrm{m}$.

b) Relative mRNA levels of IL-6, ILIA, COX-2, and $I L-10$, were assessed by RT-qPCR in the same groups as in a). ANOVA, post hoc Tukey test: $n=3$ of each treatment, * $\mathrm{p}<0.05, * * \mathrm{p}<0.05, * * * \mathrm{p}<0.001$, differences versus $\mathrm{V} ; \# \mathrm{p}<0.05$, \#\# $\mathrm{p}<0.01, \# \# \#$ $\mathrm{p}<0.001$, differences between other treatments. 
Boix FIG. 1
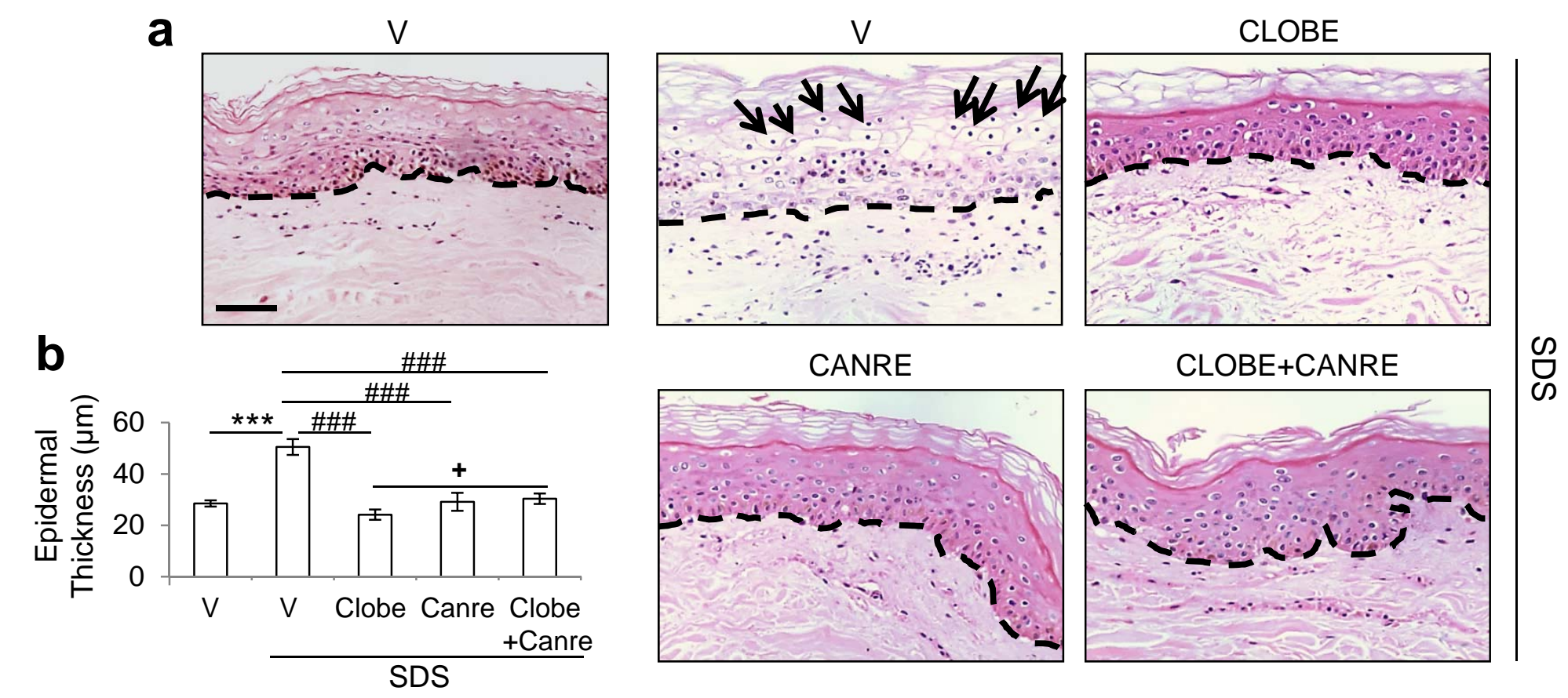


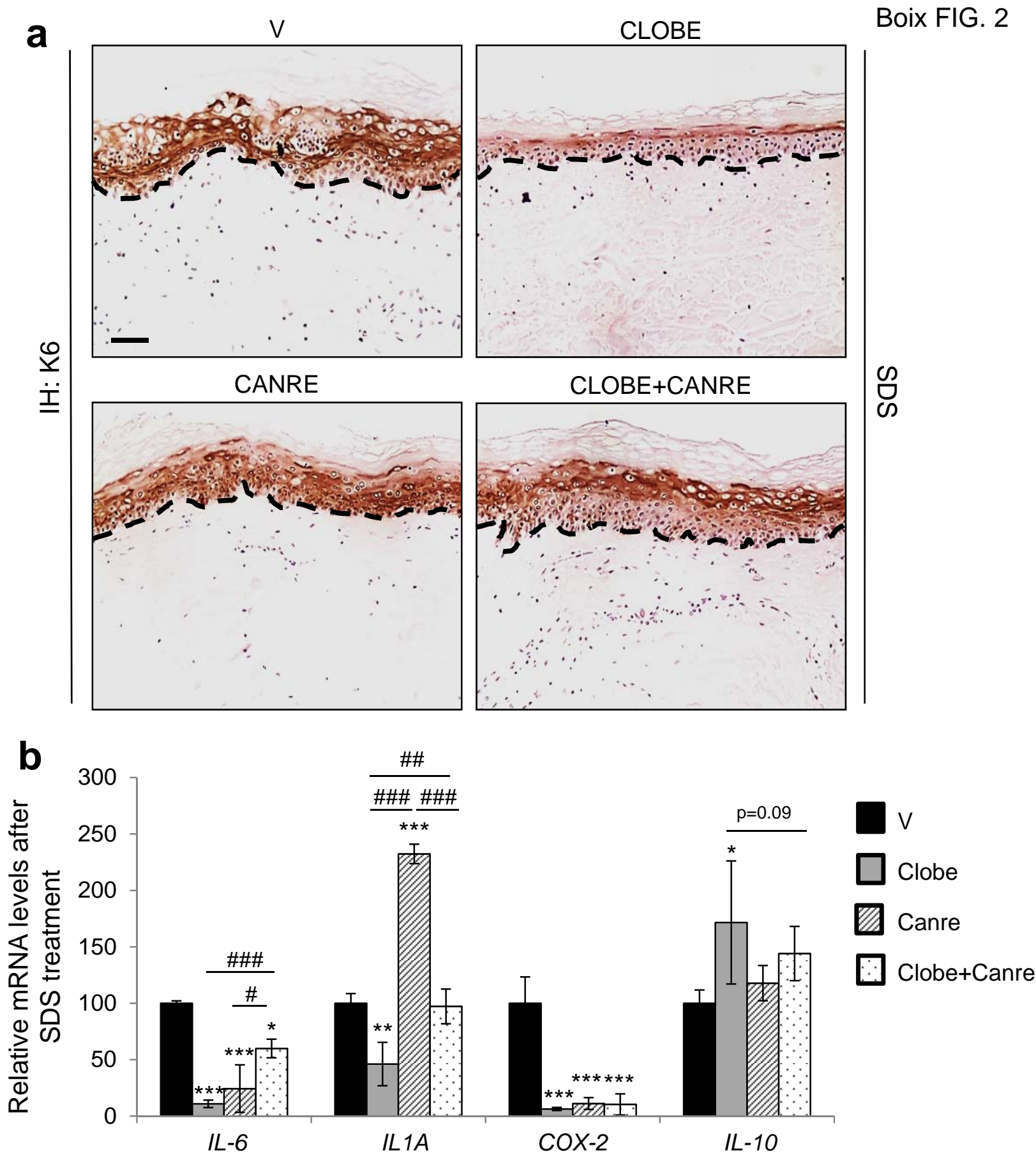




\section{Supplementary Information}

\section{Supplementary Materials and Methods}

\section{Skin samples}

Human skin was obtained from patients subjected to surgery. Patients were informed and had no objection that the skin discarded from surgery could be used for research purposes. Written patient consent was not required because French law considers that it is not mandatory for noninterventional biological collection for immunological and molecular research. This procedure was approved by the Comité de Protection des Personnes Ile de France V.

\section{Sample processing, and histological analysis}

After the treatments, each sample was divided in two parts. One was frozen at $-80^{\circ} \mathrm{C}$ for RNA isolation and RT-qPCR, and the other was fixed in $70 \%$ ethanol followed by paraffin embedding. $4 \mu \mathrm{m}$ skin sections were obtained and stained with hematoxylin-eosin, and Masson's trichrome (Sigma, St Louis, MO), or used for K6 immunohistochemistry (PRB-169P, Biolegend, San Diego, CA), as described (5). Epidermal thickness measurement was performed using the software IMAGE J (Rasband, W.S., ImageJ, U. S. National Institutes of Health, Bethesda, Maryland). At least five photomicrographs were taken of each section and at least five measurements were taken from each photomicrograph using a Leica DM1000 microscope, a Leica EC3 camera and Leica LAS EZ software (Leica Microsystems, Wetzlar, Germany).

\section{$R N A$ isolation and $R T-q P C R$}

RNA was isolated using Trizol (Thermo Fisher, Thermo Fisher Scientific, Waltham, MA) and reverse transcribed using oligo-dT and RevertAid H-minus Reverse Transcriptase (Fermentas Inc., Burlington, Canada). qPCR was conducted using specific oligonucleotides (Table 1) and FastStart Universal SYBR Green Master ROX (Roche) in an Applied Biosystems 7500 Fast real time PCR system, as described (5). Data were normalized to RPLPO expression.

Table 1. RT-qPCR primers.

\begin{tabular}{lllc} 
Gene symbol & Forward sequence $5^{\prime} \rightarrow 3^{\prime}$ & Reverse sequence $5^{\prime} \rightarrow 3^{\prime}$ & product bp \\
\hline COLIA1 & GAGGGCCAAGACGAAGACATC & CAGATCACGTCATCGCACAAC & 140 \\
$I L-6$ & GGTACATCCTCGACGGCATCT & GTGCCTCTTTGCTGCTTTCAC & 81 \\
$I L 1 A$ & TGGTAGTAGCAACCAACGGGA & ACTTTGATTGAGGGCGTCATTC & 215 \\
COX-2 & CAGAGTTGGAAGCACTCTATGG & CTGTTTTAATGAGCTCTGGATC & 306 \\
$I L-10$ & GTGGAGCAGGTGAAGAATGC & GCCACCCTGATGTCTCAGTT & 137 \\
$R P L P 0$ & AGATGCAGCAGATCCGCAT & GTTCTTGCCCATCAGCACC & 59 \\
\hline
\end{tabular}

Statistical Analysis

Experimental data were analyzed using IBM SPSS Statistics 23 software as described (5). In all graphs, mean values $\pm \mathrm{SD}$ are shown. Prior to parametric testing, data were subjected to logarithmic transformation. For comparisons among more than two experimental groups, we used the one-way ANOVA which if statistically significant was followed by a post hoc Tukey multiple comparison test. $p$ values less than 0.05 were considered statistically significant. 


\section{Supplementary Figures}

Supplementary Fig. 1. Combined use of Clobetasol and MRA Canrenoate in human skin explants improved the collagen deposition and the COL1A1 mRNA levels relative to individual treatments.

a) Masson trichromic staining of human skin explants treated with vehicle only or SDS plus vehicle (V), clobetasol (Clobe), canrenoate (Canre), or Clobe+Canre. Broken lines separate epidermis from dermis. Bar: $50 \mu \mathrm{m}$.

b) Relative COL1A1 mRNA levels were assessed by RT-qPCR in all groups in a). ANOVA, post hoc Tukey test: $\mathrm{n}=3$ of each treatment, $* \mathrm{p}<0.05, * * \mathrm{p}<0.01$, differences versus vehicle only; \# $\mathrm{p}<0.05$, \#\# $\mathrm{p}<0.01$, differences versus $\mathrm{SDS}+\mathrm{V}$. 
Boix_FIG. S1

a

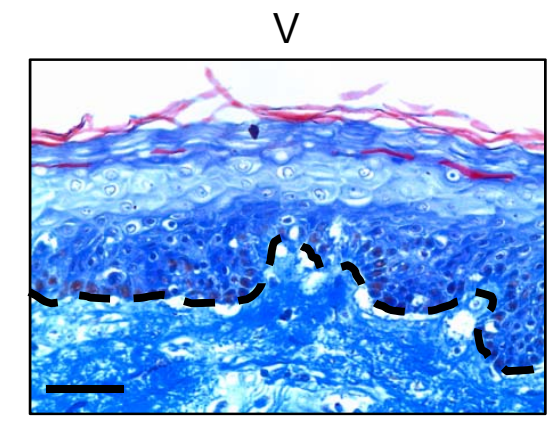

b

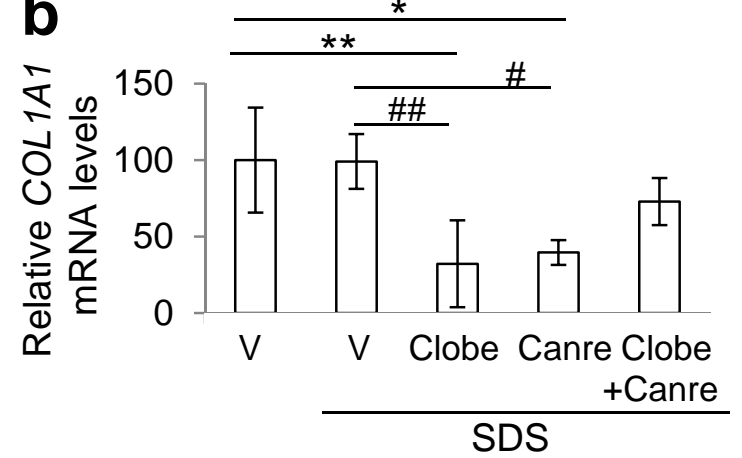

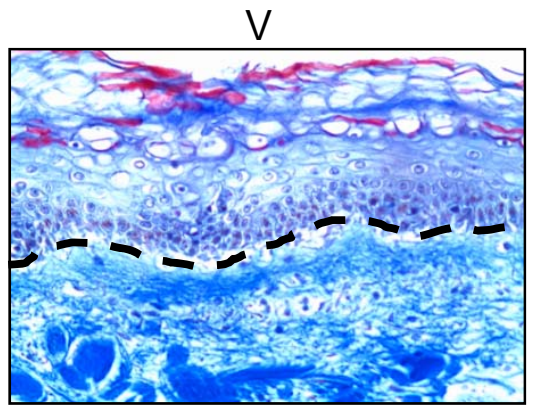

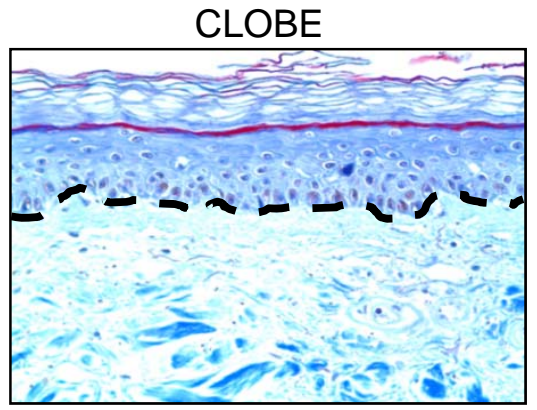

CANRE
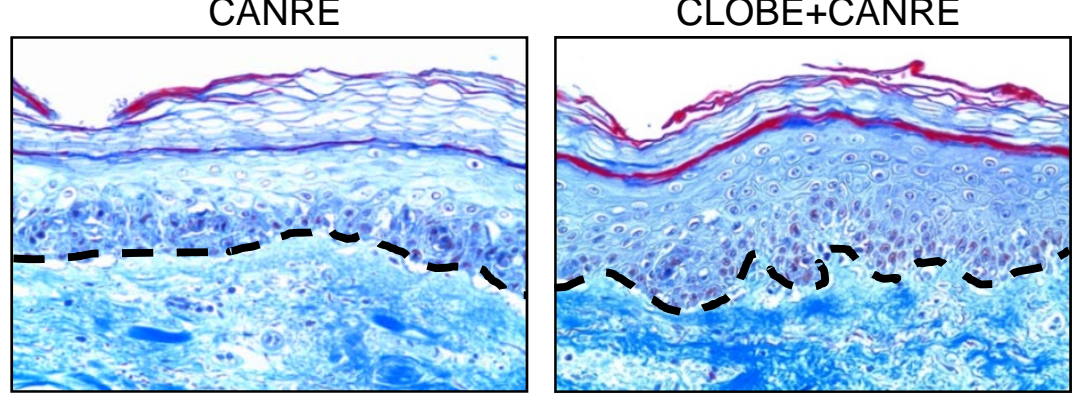

证 